\title{
Classical and quantum-linearized descriptions of degenerate optomechanical parametric oscillators
}

\author{
Sebastian Pina-Otey, ${ }^{1}$ Fernando Jiménez, ${ }^{2}$ Peter Degenfeld-Schonburg, ${ }^{3}$ and Carlos Navarrete-Benlloch ${ }^{1, *}$ * \\ ${ }^{1}$ Max-Planck-Institut für Quantenoptik, Hans-Kopfermann-strasse 1, 85748 Garching, Germany \\ ${ }^{2}$ Zentrum Mathematik der Technische Universität München, Boltzmann-strasse 3, 85747 Garching, Germany \\ ${ }^{3}$ Technische Universität München, Physik Department, James Franck Str., 85748 Garching, Germany
}

\begin{abstract}
Recent advances in the development of modern quantum technologies have opened the possibility of studying the interplay between spontaneous parametric down-conversion and optomechanics, two of the most fundamental nonlinear optical processes. Apart from practical reasons, such scenario is very interesting from a fundamental point of view, because it allows exploring the optomechanical interaction in the presence of a strongly quantum-correlated field, the spontaneously down-converted mode. In this work we analyze such problem from two approximate but valuable perspectives: the classical limit and the limit of small quantum fluctuations. We show that, in the presence of optomechanical coupling, the well-known classical phase diagram of the optical problem gets modified by the appearance of new dynamical instabilities. As for the quantum-mechanical description, we prove the ability of the squeezed down-converted field to cool down the mechanical motion not only to thermal but also to squeezed thermal mechanical states, and in a way that can be much less sensitive to parameters (e.g., detuning of the driving laser) than standard sideband cooling.
\end{abstract}

PACS numbers: 42.65.Yj,42.50.Wk,42.50.Lc,42.65.Sf

\section{INTRODUCTION}

Spontaneous parametric down-conversion is a process which occurs in crystals with second-order optical nonlinearity, where light at some frequency $2 \omega_{0}$ can be transformed into light at frequencies $\omega_{\mathrm{s}}$ and $\omega_{\mathrm{i}}$ such that $\omega_{\mathrm{s}}+\omega_{\mathrm{i}} \approx 2 \omega_{0}[1-4$. When the crystal is introduced in an optical cavity, what has the effect of enhancing the nonlinear interaction and filter the fields all at once, we obtain a so-called optical parametric oscillator (OPO), in which the down-converted field starts oscillating in the cavity only once the power of the pumping laser exceeds some threshold value (such that the nonlinear gain can compensate for the cavity losses) [1, 3, 4]. These devices have found many applications both in classical and quantum optics. In the classical case, they are among the most tunable sources of light, allowing to transform laser light into almost any (optical) frequency 1. From a quantum point of view, the down-converted photons show strong quantum correlations; particularly relevant to this work is the degenerate optical parametric oscillator (DOPO), in which down-converted photons have the same frequency $\omega_{\mathrm{s}}=\omega_{\mathrm{i}} \approx \omega_{0}$, and the corresponding output field shows nearly-perfect quadrature squeezing when working close to threshold 2 4]. Indeed, DOPOs are nowadays the sources of the highest-quality squeezed light [5] [8, which can be used to increase the sensitivity of measurements beyond the standard quantum limit 9 13, or also to generate entangled beams for quantum information purposes 14 16].

On the other hand, we have optomechanical systems, where some mechanical degree of freedom is coupled to a

\footnotetext{
* carlos.navarrete@mpq.mpg.de
}

light field via, e.g., radiation pressure [17-20]. When the interaction happens inside a cavity, the Lorentzian density of modes provided by the resonator, together with the injection of a coherent laser field with the proper detuning with respect to the cavity resonance, allows to cool down the mechanical degree of freedom to its quantum mechanical ground state through sideband cooling [21] 33. Since the mechanical degree of freedom is usually a mesoscopic system formed by many atoms, optomechanics provides a very promising platform where studying the transition from the microscopic quantum world to our natural macroscopic classical one, allowing, for example, to put bounds on collapse models 34 37. From a practical point of view, apart from offering a new platform where performing traditional quantum optical tasks such as the generation of squeezed light 38 41, transparency windows [42 46], or photon blockade effects [47, optomechanical systems might be a perfect interface between optical and microwave technologies, since mechanical degrees of freedom couple to both electromagnetic scales [48 57].

As for actual optomechanical implementations, they come out in many different forms [20: cavities with mirrors attached to cantilevers [21, 22] or in suspension 24, flexible membranes placed inside optical cavities [25, 26, 29], or localized mechanical modes in photonic crystal cavities [28, are some examples. For our current purposes, the most relevant implementations consist in (i) a whispery gallery mode resonator (a microtoroid or microdisk, for example) where light circulates around its edge via total internal reflection, pushing the whole structure, hence exciting some of its mechanical modes [23, 58, and (ii) flexible drum-shaped capacitors coupled to superconducting LC resonators [27, 44, 59].

From a fundamental point of view, the interplay between optomechanics and down-conversion seems to be 
a natural and interesting problem to study within the nonlinear quantum optics community. Recent theoretical results for the case in which the down-converted mode is seeded with an external field predict that such stimulated down-conversion process is able to enhance optomechanical cooling [60, normal mode splitting 61, and the sensitivity of mechanical quadrature measurements 62, as well as generate mechanical squeezed states 63 or even bring the optomechanical interaction to the (single-quanta) strong coupling regime 64. In a secondharmonic generation configuration (in which only the down-converted frequency is driven), multipartite optomechanical entanglement has also been predicted [65].

Modern platforms capable of combining downconversion and optomechanics in the same device have turned the motivation for studying such a scenario into a practical one. In particular, miniaturized whispering gallery mode resonators can be fabricated directly with the typical crystalline materials possessing secondorder optical nonlinearity, such that light can be downconverted while circulating on the resonator [66-78]. A completely different, but equally realistic implementation could consist on a superconducting circuit in the degenerate parametric oscillation configuration [79] coupled to a drum-shaped capacitor acting as a the mechanical degree of freedom [27, 44, 59] (see also 80, for a recent circuit QED proposal). A third and natural option would consist in using a standard OPO cavity built with a movable micromirror similar to those in [21, 22].

One of the most relevant questions in these scenarios concerns the effect that the spontaneously downconverted light, which shows strong quantum correlations, will have on the mechanical state. As a step towards understanding this question, in this work we analyze the system from a classical perspective, providing also a small glance at the quantum properties predicted by a linearization of quantum fluctuations around the classical solution. For simplicity, we stick to the degenerate case and assume that only the down-converted field is coupled to the mechanical mode. The latter is a natural situation in circuit setups (where the pump and downconverted modes are provided by different linear circuits) or when the movable mirror is introduced in an OPO that makes use of dual semi-monolithic designs [81] (see Fig. 11 to create independent cavities for the pump and down-converted fields; in the case of crystalline whispering gallery mode resonators it might require choosing a proper mechanical mode weakly coupled to the optical modes around the pump frequency. From a fundamental point of view, this configuration provides probably the most exciting scenario, since the pump stays nearcoherent for most of the DOPO parameters, and its coupling to the mechanics could mask the effects generated by the quantum-correlated down-converted field.

Concerning the classical limit, we show that, with respect the usual DOPO scenario [82 84, the most relevant effect that the optomechanical interaction has is the generation of new dynamical instabilities, as well as the

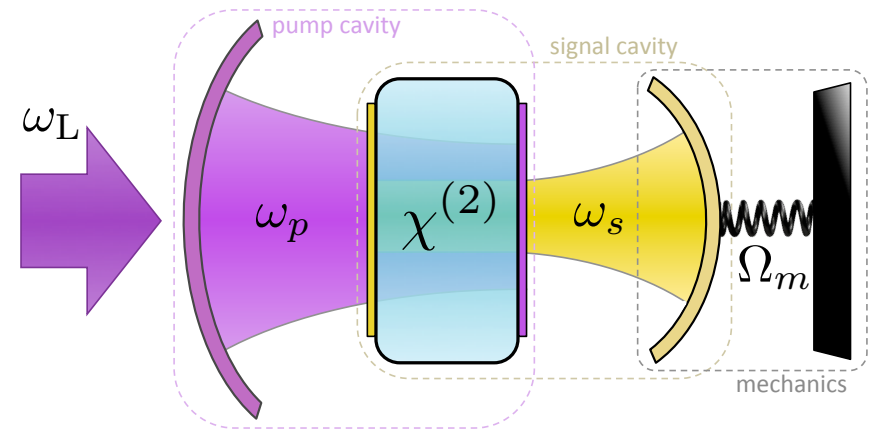

FIG. 1. Sketch of the degenerate optomechanical parametric oscillator considered in this work. It consists of a cavity containing a mode at frequency $\omega_{\mathrm{p}}$ (pump) driven by a laser at frequency $\omega_{L}$. The pump cavity shares a $\chi^{(2)}$ nonlinear crystal with another cavity containing a resonance at frequency $\omega_{\mathrm{s}}$ close to $\omega_{\mathrm{L}} / 2$ (signal), which can be then populated via degenerate down-conversion of the pumping laser in the crystal. One of the mirrors of the signal cavity is not fixed and can act then as a mechanical oscillator forced by the radiation pressure exerted from the light contained in the cavity. OPOs based on dual semi-monolithic cavities [81] can implement this exact setup, but other platforms such as crystalline whispering gallery mode resonators [66-78] or superconducting circuits [27, 44, 59, 79, 80] are already at a point where the model analyzed in our work can be studied experimentally.

modification of the region of intensity bistability. On the other hand, regarding the quantum linearized theory, we first argue how it completely fails to capture the physics below threshold, where the optomechanical interaction is purely nonlinear and requires more sophisticated techniques to describe it, which is precisely what we did in a recent work 85. Nevertheless, the linearized description provides reasonable predictions above threshold, and we use it to show that the down-converted field can have a deep impact on the mechanical state, but very different from that of the standard coherently-pumped optomechanical cavity. In particular, we show that it can cool down the mechanical motion in a way less sensitive to parameters (particularly detuning) than the traditional sideband cooling, even generating squeezed thermal mechanical states as the optomechanical interaction is increased. Let us remark that an understanding of the classical phase diagram is instrumental prior to performing a more accurate quantum analysis, as we already emphasized in Ref. [85].

The article is organized as follows. First, we introduce the model of the system, which we have called degenerate optomechanical parametric oscillator (DOMPO) and we describe through a set of quantum Langevin equations. Next we study the classical limit of the model, finding its steady states and analyzing their stability. Finally, we apply the standard linearization technique to study the quantum properties of the mechanical mode in regions of the parameter space where the classical stationary solution is stable. 


\section{THE MODEL}

Even though the actual implementation can differ from the simple picture sketched in Fig. 1, a DOMPO can be schematically understood as depicted there. Consider a cavity pumped by a laser at frequency $\omega_{\mathrm{L}}$ close to one of its resonances $\omega_{\mathrm{p}}$. This cavity (denoted by pump cavity) shares a second-order nonlinear crystal with another cavity (signal cavity) in which a resonance $\omega_{\mathrm{s}}$ close to $\omega_{\mathrm{L}} / 2$ exists, and therefore can be populated via downconversion of pump photons in the crystal. Finally, one mirror of the signal cavity can oscillate at frequency $\Omega_{\mathrm{m}}$, and acts then as a mechanical oscillator which is forced via the radiation pressure exerted by the signal field.

Let us denote by $\hat{x}_{\mathrm{m}}=\hat{Q} / Q_{0}$ the displacement of the mirror $(\hat{Q})$ normalized to the zero-point position fluctuations $Q_{0}=\sqrt{\hbar / 2 \Omega_{\mathrm{m}} M}$, where $M$ is the mirror's mass. We also normalize the corresponding momentum $\hat{P}$ to the zero-point momentum fluctuations $P_{0}=\hbar / Q_{0}$, obtaining the normalized momentum $\hat{p}_{\mathrm{m}}=\hat{P} / P_{0}$. We define annihilation operators $\hat{a}_{j}$ for pump $(j=\mathrm{p})$ and signal $(j=\mathrm{s})$ photons, with corresponding creation operators $\hat{a}_{j}^{\dagger}$. These operators satisfy the commutation relations $\left[\hat{x}_{\mathrm{m}}, \hat{p}_{\mathrm{m}}\right]=2 \mathrm{i}$, and $\left[\hat{a}_{j}, \hat{a}_{l}^{\dagger}\right]=\delta_{j l}$, any other commutator between them being zero. In a picture rotating at the laser frequency $\omega_{\mathrm{L}}$ for the pump and $\omega_{\mathrm{L}} / 2$ for the signal, the physical processes described in the previous paragraph are then captured by the Hamiltonian $\hat{H}=\hat{H}_{\mathrm{O}}+\hat{H}_{\mathrm{M}}+\hat{H}_{\mathrm{DC}}+\hat{H}_{\mathrm{OM}}$, with [2, 20]

$$
\begin{aligned}
\hat{H}_{\mathrm{O}} & =-\sum_{j=\mathrm{p}, \mathrm{s}} \hbar \Delta_{j} \hat{a}_{j}^{\dagger} \hat{a}_{j}+\mathrm{i} \hbar \mathcal{E}_{\mathrm{L}}\left(\hat{a}_{\mathrm{p}}^{\dagger}-\hat{a}_{\mathrm{p}}\right), \\
\hat{H}_{\mathrm{M}} & =\frac{\hbar \Omega_{\mathrm{m}}}{4}\left(\hat{x}_{\mathrm{m}}^{2}+\hat{p}_{\mathrm{m}}^{2}\right) \\
\hat{H}_{\mathrm{DC}} & =\mathrm{i} \hbar \chi\left(\hat{a}_{\mathrm{p}} \hat{a}_{\mathrm{s}}^{\dagger 2}-\hat{a}_{\mathrm{p}}^{\dagger} \hat{a}_{\mathrm{s}}^{2}\right), \\
\hat{H}_{\mathrm{OM}} & =-g_{\mathrm{s}} \hat{a}_{\mathrm{s}}^{\dagger} \hat{a}_{\mathrm{s}} \hat{x}_{\mathrm{m}},
\end{aligned}
$$

where $\Delta_{\mathrm{p}}=\omega_{\mathrm{L}}-\omega_{\mathrm{p}}$ and $\Delta_{\mathrm{s}}=\omega_{\mathrm{L}} / 2-\omega_{\mathrm{s}}$ denote the detuning of the laser with respect to the pump and signal modes, $\mathcal{E}_{\mathrm{L}}$ is the pump cavity's driving rate (proportional to the square root of the power of the external laser), $\chi$ is the down-conversion rate (proportional to the crystal's nonlinear susceptibility), and $g_{\mathrm{s}}$ is the opto-mechanical scattering rate (which depends strongly on the particular implementation).

In addition to these coherent processes, the system is subject to incoherent processes. In particular, we need to take into account the loss of photons through the partially transmitting mirrors (open cavities), and the coupling of the mechanical oscillator to its thermal environment, reaching some equilibrium temperature $T$ in the absence of light. We choose to include these processes at the level of the Heisenberg equations of motion, leading to the widely-used quantum Langevin equations [86, 87.

$$
\begin{aligned}
\frac{d \hat{x}_{\mathrm{m}}}{d t} & =\Omega_{\mathrm{m}} \hat{p}_{\mathrm{m}} \\
\frac{d \hat{p}_{\mathrm{m}}}{d t} & =-\gamma_{\mathrm{m}} \hat{p}_{\mathrm{m}}-\Omega_{\mathrm{m}} \hat{x}_{\mathrm{m}}+2 g_{\mathrm{s}} \hat{a}_{\mathrm{s}}^{\dagger} \hat{a}_{\mathrm{s}}+\sqrt{4 \gamma_{\mathrm{m}} \bar{n}_{\mathrm{th}}} \hat{p}_{\mathrm{m}, \mathrm{in}}(t), \\
\frac{d \hat{a}_{\mathrm{p}}}{d t} & =\mathcal{E}_{\mathrm{L}}-\left(\gamma_{\mathrm{p}}-\mathrm{i} \Delta_{\mathrm{p}}\right) \hat{a}_{\mathrm{p}}-\frac{\chi}{2} \hat{a}_{\mathrm{s}}^{2}+\sqrt{2 \gamma_{\mathrm{p}}} \hat{a}_{\mathrm{p}, \mathrm{in}}(t), \\
\frac{d \hat{a}_{\mathrm{s}}}{d t} & =-\left(\gamma_{\mathrm{s}}-\mathrm{i} \Delta_{\mathrm{s}}-\mathrm{i} g_{\mathrm{s}} \hat{x}_{\mathrm{m}}\right) \hat{a}_{\mathrm{s}}+\chi \hat{a}_{\mathrm{p}} \hat{a}_{\mathrm{s}}^{\dagger}+\sqrt{2 \gamma_{\mathrm{s}}} \hat{a}_{\mathrm{s}, \mathrm{in}}(t),
\end{aligned}
$$

where $\gamma_{j}$ are the rates of exchange of excitations of the modes with their corresponding environments, and the input operators have zero mean, $\left\langle\hat{a}_{j, \text { in }}(t)\right\rangle=\left\langle\hat{p}_{\mathrm{m}, \text { in }}(t)\right\rangle=$ $0)$, and non-zero two-time correlators

$$
\left\langle\hat{a}_{j, \text { in }}(t) \hat{a}_{j, \text { in }}^{\dagger}\left(t^{\prime}\right)\right\rangle=\left\langle\hat{p}_{\mathrm{m}, \text { in }}(t) \hat{p}_{\mathrm{m}, \text { in }}\left(t^{\prime}\right)\right\rangle=\delta\left(t-t^{\prime}\right),
$$

and play the role of the environmental quantum fluctuations driving the system. In this equations we have assumed to be working in the high-temperature limit where the number of phonons at thermal equilibrium can be approximated by $\bar{n}_{\mathrm{th}} \approx k_{B} T / \hbar \Omega_{\mathrm{m}} \gg 1$.

Before studying the equations, it is convenient to make a variable change that will allow us to see how many free parameters they really have. To this aim, we define the following normalized parameters

$$
\begin{aligned}
g_{\mathrm{DC}} & =\frac{\chi}{\sqrt{\gamma_{\mathrm{p}} \gamma_{\mathrm{s}}}}, \quad \sigma=\frac{\chi \mathcal{E}_{\mathrm{L}}}{\gamma_{\mathrm{p}} \gamma_{\mathrm{s}}}, \quad \kappa=\frac{\gamma_{\mathrm{p}}}{\gamma_{\mathrm{s}}}, \\
\delta_{j} & =\frac{\Delta_{j}}{\gamma_{j}} \quad \gamma=\frac{\gamma_{\mathrm{m}}}{\gamma_{\mathrm{s}}}, \quad \Omega=\frac{\Omega_{\mathrm{m}}}{\gamma_{\mathrm{s}}}, \quad g=\frac{g_{\mathrm{s}} / \gamma_{\mathrm{s}}}{g_{\mathrm{DC}} \sqrt{\Omega}},
\end{aligned}
$$

time $\tau=\gamma_{\mathrm{s}} t$, system operators

$$
\begin{array}{ll}
\hat{b}_{\mathrm{s}}=g_{\mathrm{DC}} \hat{a}_{\mathrm{s}}, & \hat{b}_{\mathrm{p}}=\sqrt{\kappa} g_{\mathrm{DC}} \hat{a}_{\mathrm{p}}, \\
\hat{p}=\frac{g_{\mathrm{DC}}}{\sqrt{\Omega}} \hat{p}_{\mathrm{m}}, & \hat{x}=g_{\mathrm{DC}} \sqrt{\Omega} \hat{x}_{\mathrm{m}},
\end{array}
$$

and input operators

$$
\begin{aligned}
\hat{b}_{j, \text { in }}(\tau) & =\frac{1}{\sqrt{\gamma_{\mathrm{s}}}} \hat{a}_{j, \text { in }}\left(\tau / \gamma_{\mathrm{s}}\right), \\
\hat{p}_{\mathrm{in}}(\tau) & =\frac{1}{\sqrt{\gamma_{\mathrm{s}}}} \hat{p}_{\mathrm{m}, \text { in }}\left(\tau / \gamma_{\mathrm{s}}\right),
\end{aligned}
$$

which satisfy the same correlators as before, see Eq. (3), but now with respect to the dimensionless time $\tau$. With these changes, the quantum Langevin equations are transformed into

$$
\begin{aligned}
\frac{d \hat{x}}{d \tau} & =\Omega^{2} \hat{p} \\
\frac{d \hat{p}}{d \tau} & =-\gamma \hat{p}-\hat{x}+2 g \hat{b}_{\mathrm{s}}^{\dagger} \hat{b}_{\mathrm{s}}+\sqrt{\frac{4 \gamma \bar{n}_{\mathrm{th}}}{\Omega}} g_{\mathrm{DC}} \hat{p}_{\mathrm{in}}(\tau), \\
\frac{1}{\kappa} \frac{d \hat{b}_{\mathrm{p}}}{d \tau} & =\sigma-\left(1-\mathrm{i} \delta_{\mathrm{p}}\right) \hat{b}_{\mathrm{p}}-\frac{1}{2} \hat{b}_{\mathrm{s}}^{2}+\sqrt{2} g_{\mathrm{DC}} \hat{b}_{\mathrm{p}, \text { in }}(t), \\
\frac{d \hat{b}_{\mathrm{s}}}{d \tau} & =-\left(1-\mathrm{i} \delta_{\mathrm{s}}-\mathrm{i} g \hat{x}\right) \hat{b}_{\mathrm{s}}+\hat{b}_{\mathrm{p}} \hat{b}_{\mathrm{s}}^{\dagger}+\sqrt{2} g_{\mathrm{DC}} \hat{b}_{\mathrm{s}, \text { in }}(t) .
\end{aligned}
$$


It is interesting to note that $g$ basically provides the ratio between the single-photon optomechanical and downconversion couplings, and hence, assuming $\sqrt{\Omega}$ to be of order 1 , they inform us about which of the two nonlinear processes dominates.

The quantum langevin equations are nonlinear operator equations, and hence it is a formidable task to obtain results directly from them without further approximations. In the following, we analyze two relevant limits of these equations: the classical limit and the limit of 'small' quantum fluctuations.

\section{CLASSICAL ANALYSIS}

The classical limit of the model is obtained by assuming that all the modes of the system are in a coherent state. Defining the corresponding amplitudes $\beta_{j}=\left\langle\hat{b}_{j}\right\rangle$, $x=\langle\hat{x}\rangle$, and $p=\langle\hat{p}\rangle$, and taking the expectation value of the quantum Langevin equations (7), such approximation leads to the classical equations

$$
\begin{aligned}
\dot{x} & =\Omega^{2} p, \\
\dot{p} & =-\gamma p-x+2 g\left|\beta_{\mathrm{s}}\right|^{2}, \\
\kappa^{-1} \dot{\beta}_{\mathrm{p}} & =\sigma-\left(1-\mathrm{i} \delta_{\mathrm{p}}\right) \beta_{\mathrm{p}}-\beta_{\mathrm{s}}^{2} / 2, \\
\dot{\beta}_{\mathrm{s}} & =-\left(1-\mathrm{i} \delta_{\mathrm{s}}-\mathrm{i} g x\right) \beta_{\mathrm{s}}+\beta_{\mathrm{p}} \beta_{\mathrm{s}}^{*} .
\end{aligned}
$$

As non-trivial nonlinear equations, it is not possible to find their time-dependent analytical solutions other than numerically. However, working with a dissipative system, we are mainly interested in its behavior for long times (asymptotic limit), and there is a lot that we can say about this without really solving the full nonlinear equations; in particular, we follow closely the procedure already applied to detuned DOPOs 8284 .

\section{A. Stationary solutions}

The simplest asymptotic behavior that one can expect is that the system reaches some steady state. Hence, it is always convenient to start by finding the timeindependent solutions to the nonlinear equations, which we denote by a bar, e.g., $\bar{x}$; when needed, we will write the complex field amplitudes as $\bar{\beta}_{j}=\sqrt{I_{j}} \exp \left(\mathrm{i} \varphi_{j}\right)$, with real variables $\left.\left.\varphi_{j} \in\right]-\pi, \pi\right]$ and $I_{j} \geq 0$.

All the stationary solutions of $(8)$ have $\bar{p}=0$ and $\bar{x}=$ $2 g I_{\mathrm{s}}$, leaving us with

$$
\begin{aligned}
\sigma & =\left(1-\mathrm{i} \delta_{\mathrm{p}}\right) \bar{\beta}_{\mathrm{p}}+\bar{\beta}_{\mathrm{s}}^{2} / 2, \\
\bar{\beta}_{\mathrm{p}} \bar{\beta}_{\mathrm{s}}^{*} & =\left(1-\mathrm{i} \delta_{\mathrm{s}}-2 \mathrm{i} g^{2} I_{\mathrm{s}}\right) \bar{\beta}_{\mathrm{s}} .
\end{aligned}
$$

We distinguish then two types of stationary solutions: trivial or below-threshold solutions, which have $I_{\mathrm{s}}=0$, and nontrivial or above-threshold solutions with $I_{\mathrm{s}} \neq 0$.

In the trivial case, the solution is simply

$$
\bar{\beta}_{\mathrm{s}}=0 \quad \text { and } \quad \bar{\beta}_{\mathrm{p}}=\sigma /\left(1-\mathrm{i} \delta_{\mathrm{p}}\right) .
$$

As for the nontrivial solutions, we find their analytic expression as follows. First, note that $\left(9 \mathrm{~b}\right.$ implies $\bar{\beta}_{\mathrm{p}}=$ $\left(1-\mathrm{i} \delta_{\mathrm{s}}-2 \mathrm{i} g^{2} I_{\mathrm{s}}\right) e^{2 \mathrm{i} \varphi_{\mathrm{s}}}$, which plugged into 9a) leads to $e^{-2 \mathrm{i} \varphi_{\mathrm{s}}} \sigma=\left(1-\mathrm{i} \delta_{\mathrm{p}}\right)\left(1-\mathrm{i} \delta_{\mathrm{s}}-2 \mathrm{i} g^{2} I_{\mathrm{s}}\right)+I_{\mathrm{s}} / 2$, whose absolute value squared gives us a second order polynomial for the signal intensity

$$
\begin{aligned}
\sigma^{2} & =\left[1+\frac{I_{\mathrm{s}}}{2}-\delta_{\mathrm{p}}\left(\delta_{\mathrm{s}}+2 g^{2} I_{\mathrm{s}}\right)\right]^{2}+\left(\delta_{\mathrm{p}}+\delta_{\mathrm{s}}+2 g^{2} I_{\mathrm{s}}\right)^{2} \\
& \equiv q_{0}+q_{1} I_{\mathrm{s}}+q_{2} I_{\mathrm{s}}^{2}
\end{aligned}
$$

with

$$
\begin{aligned}
& q_{0}=\left(1+\delta_{\mathrm{p}}^{2}\right)\left(1+\delta_{\mathrm{s}}^{2}\right), \\
& q_{1}=\left(1-\delta_{\mathrm{p}} \delta_{\mathrm{s}}\right)+4 \delta_{\mathrm{s}}\left(1+\delta_{\mathrm{p}}^{2}\right) g^{2}, \\
& q_{2}=4 g^{4}+\left(\frac{1}{2}-2 \delta_{\mathrm{p}} g^{2}\right)^{2} .
\end{aligned}
$$

Depending on the value of the parameters, this equation can have a single real positive solution or two, as shown in Fig. 2. In order to find for which values of the parameters (in particular of the injection $\sigma$ and the detunings $\delta_{j}$, experimentally tunable) this happens, we just need to obtain the expression for the turning point, marked as TP in Fig. 2, which is nothing but the extremum of $\sigma^{2}\left(I_{\mathrm{s}}\right)$, that is,

$$
\left.\frac{\partial \sigma^{2}}{\partial I_{\mathrm{s}}}\right|_{I_{\mathrm{s}}=I_{\mathrm{s}}^{\mathrm{TP}}}=0 \quad \Longrightarrow \quad I_{\mathrm{s}}^{\mathrm{TP}}=-q_{1} / 2 q_{2} .
$$

Taking into account that $q_{2}>0$, the turning point will exist only if $q_{1}<0$, which gives us a condition on the detunings for a given optomechanical coupling:

$$
\delta_{\mathrm{p}} \delta_{\mathrm{s}}>1+4 \delta_{\mathrm{s}}\left(1+\delta_{\mathrm{p}}^{2}\right) g^{2} .
$$

Hence, when this condition is satisfied, we will have two possible steady-state signal intensities (three counting the trivial one) for injections $\left.\left.\sigma^{2} \in\right] q_{0}-q_{1}^{2} / 4 q_{2}, q_{0}\right]$, see Fig. 2k. Let us anticipate, however, that the branch connecting the trivial solution with the upper branch of the nontrivial one is unstable (see Fig. 2), so only two out of the three possible solutions can be observed in real experiments, leading to an intensity bistability common in nonlinear optical systems. Finally, notice that condition (14) with $g=0$ is in agreement with that found for detuned DOPOs 82,84 .

\section{B. Linear stability analysis}

The existence of a mathematical solution of the nonlinear equations is not enough to ensure its physical reality: it also needs to be stable against perturbations, since in the real world these are unavoidable, and therefore we would never be able to observe the system in the corresponding solution otherwise. Hence, in the following we proceed to study the stability of the stationary solutions found above. 


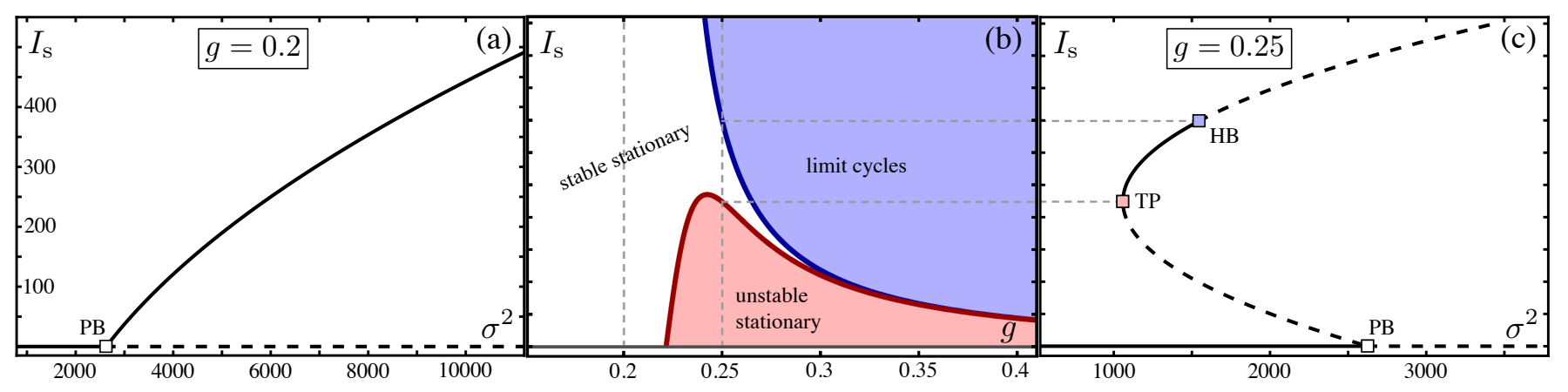

FIG. 2. We show the bifurcations and corresponding stable and unstable regions for one particular example for which we have chosen $\gamma=0.005, \Omega=-\delta_{\mathrm{s}}=10, \delta_{\mathrm{p}}=5$, and $\kappa=100$, the first three being typical parameters when aiming for sideband cooling in optomechanical systems. Note that this fixes all the parameters but $g$ and $I_{\mathrm{s}}$ (we are using the steady-state intensity $I_{\mathrm{s}}$ as a parameter instead of $\sigma$, because the latter can be uniquely determined from the former, but in general not the other way around). In (b) we show in the space of these parameters the turning point (red thick line) and the single Hopf instability (blue thick line) found in this example, coloring the regions where they make the stationary solution unstable. Note that for this choice of parameters, in the absence of optomechanical coupling there are no instabilities apart from the trivial pitchfork bifurcation, as can be checked from conditions (14) and (17). Hence, we see that the effect of the optomechanical coupling in this case consists in introducing new instabilities which greatly reduce the domain of stabity of the nontrivial stationary state. In (a) and (c) we show how the steady-state intensity $I_{\mathrm{S}}$ depends on the injection $\sigma^{2}$ for two specific values of $g$, corresponding to the vertical grey dashed lines in the parameter space (b), denoting its unstable and stable regions by dashed and solid lines, respectively. In (a) we have chosen $g=0.2$, for which no instabilities are present in the nontrivial solution as can be appreciated in (b), and hence only the pitchfork instability connecting the trivial and nontrivial solutions is present. In (c), on the other hand, we have chosen $g=0.25$, for which we find both a turning point (and hence a domain of bistability between the trivial and nontrivial solutions) and a Hopf bifurcation leading to time-dependent asymptotic solutions.

Let us collect the variables of the system in a vector $\mathbf{b}=\operatorname{col}\left(x, p, \beta_{\mathrm{p}}, \beta_{\mathrm{p}}^{*}, \beta_{\mathrm{s}}, \beta_{\mathrm{s}}^{*}\right)$. The stability of a given stationary solution $\overline{\mathbf{b}}$ can be analyzed as follows 88 . We consider small fluctuations around it by writing $\mathbf{b}(t)=$ $\overline{\mathbf{b}}+\delta \mathbf{b}(t)$, introduce this ansatz into the nonlinear system (8), and keep only terms which are linear in the fluctu- ations, obtaining a linear system $\delta \dot{\mathbf{b}}=\mathcal{L} \delta \mathbf{b}$, where $\mathcal{L}$ is the so-called linear stability matrix. This matrix depends on the system parameters and the particular stationary solution whose stability we are considering, and in our case is given by

$$
\mathcal{L}=\left(\begin{array}{cccccc}
0 & \Omega^{2} & 0 & 0 & 0 & 0 \\
-1 & -\gamma & 0 & 0 & 2 g \bar{\beta}_{\mathrm{s}}^{*} & 2 g \bar{\beta}_{\mathrm{s}} \\
0 & 0 & -\kappa\left(1-\mathrm{i} \delta_{\mathrm{p}}\right) & 0 & -\kappa \bar{\beta}_{\mathrm{s}} & 0 \\
0 & 0 & 0 & -\kappa\left(1+\mathrm{i} \delta_{\mathrm{p}}\right) & 0 & -\kappa \bar{\beta}_{\mathrm{s}}^{*} \\
\mathrm{i} g \bar{\beta}_{\mathrm{s}} & 0 & \bar{\beta}_{\mathrm{s}}^{*} & 0 & -\left(1-\mathrm{i} \delta_{\mathrm{s}}-\mathrm{i} g \bar{x}\right) & \bar{\beta}_{\mathrm{p}} \\
-\mathrm{i} g \bar{\beta}_{\mathrm{s}}^{*} & 0 & 0 & \bar{\beta}_{\mathrm{s}} & \bar{\beta}_{\mathrm{p}}^{*} & -\left(1+\mathrm{i} \delta_{\mathrm{s}}+\mathrm{i} g \bar{x}\right)
\end{array}\right)
$$

Since the equation for the fluctuations is linear, it is then clear that their dynamical behaviour is controlled by the eigenvalues of this matrix. In particular, the fluctuations will be damped and disappear in the asymptotic limit only if the real part of all the eigenvalues is negative. Hence, we say that a stationary solution $\overline{\mathbf{b}}$ is stable (and therefore physical) when all the eigenvalues of its corresponding linear stability matrix $\mathcal{L}(\overline{\mathbf{b}})$ have negative real part.

The points in the parameter space where at least one of the eigenvalues has zero real part are known as critical points, instabilities, or bifurcations, and they separate the regions in which the stationary solution changes from sta- ble to unstable. We can distinguish two types of instabilities: pitchfork or static bifurcations, where the imaginary part of the relevant eigenvalue is also zero, which connect the stationary solution with another stationary solution; and Hopf or dynamic bifurcations, where the imaginary part of the relevant eigenvalue is non-zero, which connect the stationary solution with a time-dependent solution (usually some periodic solution, known in this context as a periodic orbit or limit cycle).

Before proceeding, let us comment on one subtle point concerning the system parameters. The linear stability matrix 15) does not depend explicitly on the injection $\sigma$, it does only implicitly through the intracavity stationary 
amplitudes $\bar{\beta}_{\mathrm{p}}$ and $\bar{\beta}_{\mathrm{s}}$. It is then convenient to use either $I_{\mathrm{p}}$ or $I_{\mathrm{s}}$ as a parameter instead of $\sigma$ when dealing with the trivial or nontrivial solutions, respectively, knowing that $\sigma$ can always be uniquely determined from them by using (10) or (11).

Let us now proceed to discuss the instabilities that can be found in the DOMPO. We provide here a summary of the main results, and leave the detailed derivations for Appendix A Concerning the trivial solution, it possess only one bifurcation appearing when $I_{\mathrm{p}}=1+\delta_{\mathrm{s}}^{2}$. The trivial solution becomes unstable for $I_{\mathrm{p}}>1+\delta_{\mathrm{s}}^{2}$, or in terms of the injection, when $\sigma^{2}>\left(1+\delta_{\mathrm{s}}^{2}\right)\left(1+\delta_{\mathrm{p}}^{2}\right)$. Note that this is precisely the point at which the trivial and nontrivial solutions coalesce, see the points marked as PB in Fig. 2, and hence this pitchfork bifurcation simply connects these two stationary solutions. As for the nontrivial solution, it provides one more static instability at $I_{\mathrm{s}}=I_{\mathrm{s}}^{\mathrm{TP}}$. Hence, we see that the turning point of the nontrivial solution is an instability, and it is simple to check that the lower branch of the nontrivial solution connecting the upper branch with the trivial solution is unstable (for example by evaluating the eigenvalues numerically for one set of parameters), as shown in Fig. 2 . In other words, the turning point is a pitchfork bifurcation connecting the unstable lower branch with the upper branch, which is stable in all its domain of existence, except for possible Hopf bifurcations which we discuss next.

The behaviour of the dynamical instabilities of the nontrivial solution is very rich in the DOMPO. Let us first note that in the absence of optomechanical coupling $(g=0)$ there is a single Hopf bifurcation located at (see Appendix $\mathrm{A}$ and 82 [84])

$$
I_{\mathrm{s}}^{\mathrm{HB}}=-\frac{\left(1+\delta_{\mathrm{p}}^{2}\right)\left[(2+\kappa)^{2}+\kappa^{2} \delta_{\mathrm{p}}^{2}\right]}{(1+\kappa)^{2}\left(2+\kappa+\kappa \delta_{\mathrm{p}}^{2}+2 \delta_{\mathrm{p}} \delta_{\mathrm{s}}\right)},
$$

which requires

$$
\delta_{\mathrm{p}} \delta_{\mathrm{s}}<-1-\kappa\left(1+\delta_{\mathrm{p}}^{2}\right) / 2,
$$

to exist (otherwise $I_{\mathrm{s}}^{\mathrm{HB}}<0$ ), which incidentally means that it does not exist when there is bistability in the system (what requires $\delta_{\mathrm{p}} \delta_{\mathrm{s}}>1$ ). It is possible to show that the portion of the nontrivial solution with $I_{\mathrm{s}}>I_{\mathrm{s}}^{\mathrm{HB}}$ becomes unstable, and the limit cycles become chaotic for large enough injections 82 84. The main effect of optomechanics, that is, of increasing $g$, is both changing the location of this dynamical instability already present for $g=0$, as well as creating new ones that cannot be understood as a deformation of the latter. This is what we show in Fig. 2 $\mathrm{b}$ for one example, where we plot the signal intensity of the Hopf instability that we have found as a function of $g$. Let us remark that in the $g \neq 0$ case the complicated form of the linear stability matrix has prevented us from finding simple analytic expressions for such instabilities, but we have been able to find a simple way to compute them efficiently with the help of symbolic programs that has allowed us to perform an exhaustive analysis, see Appendix A

\section{QUANTUM PROPERTIES WITHIN THE LINEARIZED DESCRIPTION}

In order to characterize the quantum properties of the DOMPO, we now apply the widely-used method of standard linearization [89 92. In this approach, one assumes that the asymptotic classical solution of the system is a strong attractor, and hence quantum mechanics acts just as strongly damped fluctuations or noise driving continuously the system and trying to bring it out of equilibrium. As we did in the classical analysis, let us collect the fundamental operators of the system into a vector $\hat{\mathbf{b}}=\operatorname{col}\left(\hat{x}, \hat{p}, \hat{b}_{\mathrm{p}}, \hat{b}_{\mathrm{p}}^{\dagger}, \hat{b}_{\mathrm{s}}, \hat{b}_{\mathrm{s}}^{\dagger}\right)$. In this scenario, it is then convenient to write the operators as $\hat{\mathbf{b}}(\tau)=\overline{\mathbf{b}}+\delta \hat{\mathbf{b}}(\tau)$, where we remind that $\overline{\mathbf{b}}$ is the stationary solution found for the operators within the classical description of the system (we will not consider periodic orbits in this work, although they can also be studied with a similar approach). Assuming that $\overline{\mathbf{b}}$ is a strong classical attractor, that is, that the eigenvalues of its associated linear stability matrix $\mathcal{L}$ are large enough for quantum fluctuations to be strongly damped, one can neglect terms of the quantum Langevin equations (7) beyond linear order in the fluctuations $\delta \hat{\mathbf{b}}$, turning them into the linear system

$$
\frac{d}{d \tau} \delta \hat{\mathbf{b}}=\mathcal{L} \delta \hat{\mathbf{b}}+\sqrt{2} g_{\mathrm{DC}} \widehat{\mathbf{f}}(\tau),
$$

where we have defined the input-vector operator

$$
\widehat{\mathbf{f}}(\tau)=\operatorname{col}\left(0, \sqrt{\frac{2 \gamma \bar{n}_{\mathrm{th}}}{\Omega}} \hat{p}_{\mathrm{in}}, \hat{b}_{\mathrm{p}, \text { in }}, \hat{b}_{\mathrm{p}, \text { in }}^{\dagger}, \hat{b}_{\mathrm{s}, \text { in }}, \hat{b}_{\mathrm{s}, \text { in }}^{\dagger}\right),
$$

which acts precisely as a quantum force continuously driving the system out of equilibrium. Note that the two-time input correlators can be written in the compact form $\left\langle\hat{f}_{j}(\tau) \hat{f}_{l}\left(\tau^{\prime}\right)\right\rangle=M_{j l} \delta\left(\tau-\tau^{\prime}\right)$, where $M_{j l}$ are the elements of the matrix $\mathcal{M}=\mathcal{M}_{\mathrm{m}} \oplus \mathcal{M}_{\mathrm{p}} \oplus \mathcal{M}_{\mathrm{s}}$ with

$$
\begin{aligned}
\mathcal{M}_{\mathrm{m}} & =\left(\begin{array}{lc}
0 & 0 \\
0 & 2 \gamma \bar{n}_{\mathrm{th}} / \Omega
\end{array}\right), \\
\mathcal{M}_{\mathrm{p}} & =\left(\begin{array}{ll}
0 & \kappa \\
0 & 0
\end{array}\right), \\
\mathcal{M}_{\mathrm{s}} & =\left(\begin{array}{ll}
0 & 1 \\
0 & 0
\end{array}\right) .
\end{aligned}
$$

In the following, we will particularize these linearized quantum Langevin equations to the two types of classical stable stationary solutions that we have found (trivial and nontrivial), analyzing the behavior that they predict for the mechanical mode. From the previous discussion, it is clear that such a linearized description will break down close to the critical points of the classical theory, but as proven again and again in many nonlinear optical systems, its predictions usually provide the correct tendency of observables as one approaches the critical points, at the very least qualitatively. There are however some exceptions, corresponding to cases in which the quantum 
mechanical effects are purely nonlinear, so that linearization completely misses them. Indeed, we shall see one of such examples now.

\section{A. Failure of the method below threshold}

The first interesting thing to note about the linearization approach is how it completely fails to capture any optomechanical phenomena that might be occurring below threshold, that is, when the signal field is switched off classically, so that there is no coherent background in the mechanical and signal modes. This is clearly seen from the fact that, as we show in Sec. A 1 of Appendix A the linear stability matrix is written as a direct sum of matrices acting on each mode, so that the equations for the mechanical fluctuations $\delta \hat{x}=\hat{x}$ and $\delta \hat{p}=\hat{p}$ simply read

$$
\begin{aligned}
& \frac{d \hat{x}}{d \tau}=\Omega^{2} \hat{p}, \\
& \frac{d \hat{p}}{d \tau}=-\gamma \hat{p}-\hat{x}+\sqrt{\frac{4 \gamma \bar{n}_{\mathrm{th}}}{\Omega}} g_{\mathrm{DC}} \hat{p}_{\mathrm{in}}(\tau) .
\end{aligned}
$$

These equations receive absolutely no information from the optical modes, in particular corresponding to a harmonic oscillator in thermal equilibrium with its environment. In the same way, the fluctuations of the optical mode $\delta \hat{b}_{\mathrm{s}}=\hat{b}_{\mathrm{s}}$ are completely unaffected by the mechanics, since they obey the usual below-threshold DOPO dynamics,

$$
\frac{d \hat{b}_{\mathrm{s}}}{d \tau}=\left(-1+\mathrm{i} \delta_{\mathrm{s}}\right) \hat{b}_{\mathrm{s}}+\bar{\beta}_{\mathrm{p}} \hat{b}_{\mathrm{s}}^{\dagger}+\sqrt{2} g_{\mathrm{DC}} \hat{b}_{\mathrm{s}, \text { in }}(\tau) .
$$

As is well known [2, 3, 86, the latter equations predict that the signal mode gets more and more squeezed as the critical point of the below threshold solution $I_{\mathrm{p}}=1+\delta_{\mathrm{s}}^{2}$ is approached, denoted by $\mathrm{PB}$ in Figs. $2 \mathrm{a}$ and 2 . In fact, the linearized description predicts an infinite photon number exactly at threshold [90, 92, which of course gets regularized once more accurate approaches are used [89. 93 101. In the light of this insight, it is hard to believe that despite the large number of photons present in the signal mode, optomechanical scattering will have no effect whatsoever on the fluctuations of the modes, as Eqs. 21) and (22) predict, and one has to conclude that the linearization simply fails to capture whatever phenomena occurs below threshold. In fact, in this regime the signal photons scattered by the mechanical mode are purely quantum mechanical, with no coherent or classical background, and this is precisely what makes the optomechanical interaction $\hat{a}_{\mathrm{s}}^{\dagger} \hat{a}_{\mathrm{s}} \hat{x}_{\mathrm{m}}$ purely nonlinear or nongaussian, which is ultimately the reason why any effect related to it is completely lost upon linearization.

More sophisticated linearized descriptions such as the self-consistent linearization introduced in 89 cannot work either, since it is equivalent to making a Gaussian ansatz for the full state of the system, while the optomechanical interaction becomes purely nongaussian below threshold as we have argued above. Hence, more elaborated techniques are required below threshold, such as a numerical simulation based on the positive $\mathrm{P}$ representation [4, 93 95, 102] or the self-consistent Mori Projector operator (c-MoP) theory [101, 103], which are beyond the scope of this work, and we indeed explore in other works 85. Nevertheless, as we are about to see, standard linearization can still be a useful tool allowing us to analyze the system above threshold, and find indications of interesting phenomena.

\section{B. Above-threshold predictions}

The situation is rather different above threshold. In this case all the modes have a classical background, and hence it is possible to linearize the optomechanical interaction without loosing it completely. Consequently, it is to be expected that, even though the method will fail at the critical points of the classical theory, it will provide us with a good qualitative picture of the trend that the quantum properties of the system follow.

As explained above, the linearization method is equivalent to making a Gaussian ansatz [14 16] for the state of the quantum fluctuations around the classical solution 89, 101. Hence, within this approach the quantum properties of the system are completely characterized by the covariance matrix of all the modes $[14-16]$. Given the position $\hat{x}_{j}=\hat{a}_{j}^{\dagger}+\hat{a}_{j}$ and momentum $\hat{p}_{j}=\mathrm{i}\left(\hat{a}_{j}^{\dagger}-\hat{a}_{j}\right)$ quadratures of the optical modes $(j=\mathrm{p}, \mathrm{s})$, and defining the quadrature vector operator $\hat{\mathbf{r}}=\operatorname{col}\left(\hat{x}_{\mathrm{m}}, \hat{p}_{\mathrm{m}}, \hat{x}_{\mathrm{p}}, \hat{p}_{\mathrm{p}}, \hat{x}_{\mathrm{s}}, \hat{p}_{\mathrm{s}}\right)$, the covariance matrix $V$ is defined as the symmetric matrix with elements $V_{j l}=\left\langle\delta \hat{r}_{j} \delta \hat{r}_{l}+\delta \hat{r}_{l} \delta \hat{r}_{j}\right\rangle / 2$ [14 16]. In Appendix $B$ we explain how this object can be efficiently evaluated numerically for any value of the parameters directly from the linearized Langevin equations (18), specifically from the eigensystem of the linear stability matrix 15.

On the other hand, as explained in the introduction, the main question that we would like to explore is the effect that the optomechanical interaction has on the mechanical state. In the following we show through a set of examples how the squeezed down-converted field is able to cool down the mechanical motion. Moreover, it does so in a way that can be much less sensitive to parameters than standard sideband cooling. Furthermore, apart from cooling it, the example will show a trend of the optomechanical interaction to squeeze the thermal mechanical motion.

In order to show this, we first find the reduced state of the mechanical mode. Since within the linearized picture the state of the whole system is Gaussian, the reduced mechanical state is Gaussian as well, with a covariance matrix given by the corresponding submatrix of the full 

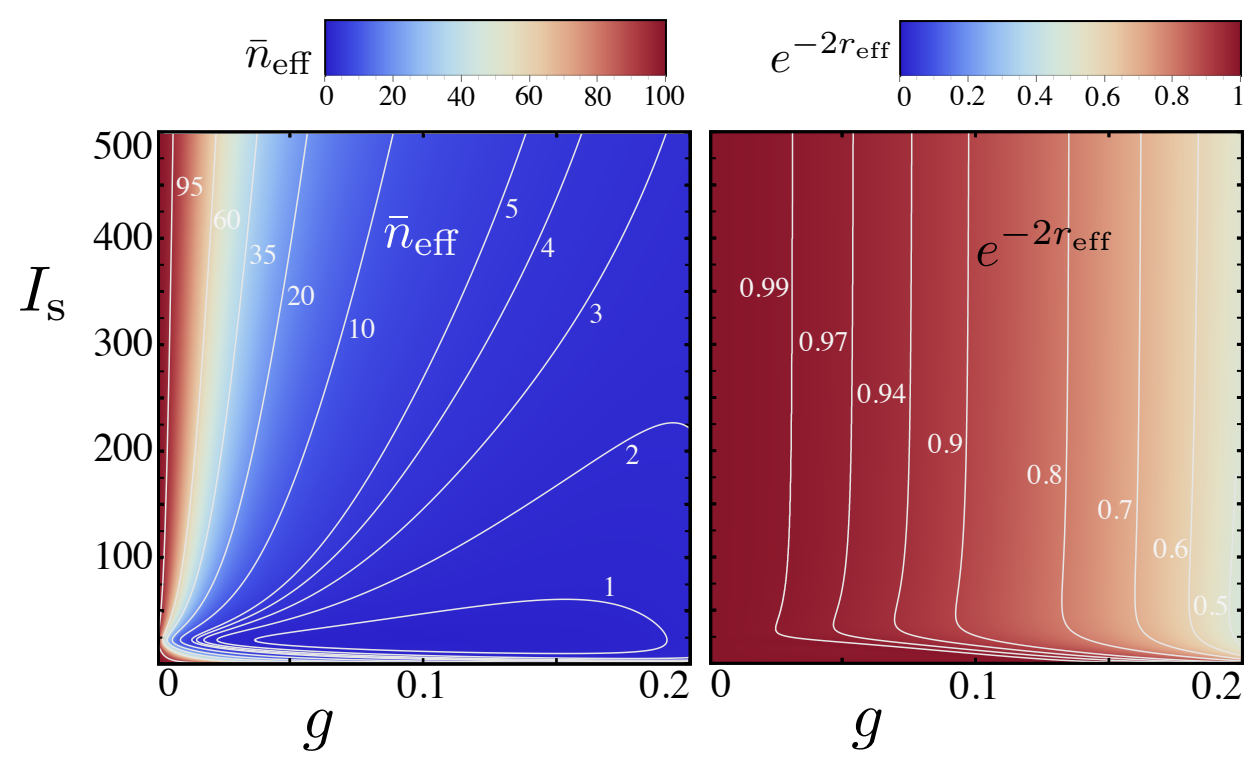

FIG. 3. Density plots of the effective phonon number $\bar{n}_{\text {eff }}$ and mechanical squeezing $e^{-2 r_{\text {eff }}}$ as a function of the optomechanical coupling $g$ and the intracavity signal intensity $I_{\mathrm{s}}$. The rest of parameters have the same values as in Fig. 2, and we assume a phonon number $\bar{n}_{\text {th }}=100$ at thermal equilibrium. Note how the mechanical state is strongly affected by the optomechanical interaction, which allows both for cooling and squeezing the mechanical motion.

covariance matrix (B4] [16]:

$$
V_{\mathrm{m}}=\left(\begin{array}{ll}
V_{11} & V_{12} \\
V_{21} & V_{22}
\end{array}\right)
$$

In order to get a better physical picture of the mechanical state, we further exploit the fact that any single-mode Gaussian state can be written as a squeezed thermal state up to a rotation in phase space [15, 16] (which simply provides the direction of phase space along which squeezing occurs). This means that the mechanical state $\hat{\rho}_{\mathrm{m}}$ can be written in the form

$$
\hat{R}(\theta) \hat{\rho}_{\mathrm{m}} \hat{R}^{\dagger}(\theta)=\hat{S}\left(r_{\mathrm{eff}}\right) \hat{\rho}_{\mathrm{th}}\left(\bar{n}_{\mathrm{eff}}\right) \hat{S}^{\dagger}\left(r_{\mathrm{eff}}\right) \equiv \tilde{\rho}_{\mathrm{m}},
$$

for some phase-shift operator $\hat{R}(\theta)=\exp \left[\mathrm{i} \theta\left(\hat{x}_{\mathrm{m}}^{2}+\hat{p}_{\mathrm{m}}^{2}\right) / 4\right]$, where $\hat{\rho}_{\text {th }}\left(\bar{n}_{\text {eff }}\right)$ is a thermal state with effective phonon number $\bar{n}_{\text {eff }}$, and $\hat{S}\left(r_{\text {eff }}\right)=\exp \left[i r_{\text {eff }} \hat{x}_{\mathrm{m}} \hat{p}_{\mathrm{m}} / 2\right]$ is a squeezing operator with effective squeezing parameter $r_{\text {eff }}$. We are in particular interested in the effective phonon number $\bar{n}_{\text {eff }}$ and the effective mechanical squeezing $e^{-2 r_{\text {eff }}}$, which can be written in terms of the elements of the covariance matrix as (see Appendix B

$$
\bar{n}_{\mathrm{eff}}=\left(\sqrt{V_{+} V_{-}}-1\right) / 2 \text { and } e^{-2 r_{\mathrm{eff}}}=\sqrt{V_{-} / V_{+}},
$$

where $V_{\mp}=\operatorname{tr}\left\{V_{\mathrm{m}}\right\} / 2 \mp \sqrt{\operatorname{tr}\left\{V_{\mathrm{m}}\right\}^{2} / 4-\operatorname{det}\left\{V_{\mathrm{m}}\right\}}$ are the eigenvalues of the mechanical covariance matrix. Let us now pass to analyze these parameters for some specific situation.

We take as an example the parameters of Fig. 2, for which we already presented the stable and unstable regions of the classical stationary solution. In Fig. 3 we show the variation of the effective thermal phonon number $\bar{n}_{\text {eff }}$ and squeezing $e^{-2 r_{\text {eff }}}$ with $g$, as we move above threshold, that is, $I_{\mathrm{s}}>0$. It can be appreciated how the mechanical state is deeply affected by the optomechanical interaction. In particular, we see that the effective phonon number can decrease to low values, what shows the ability of the down-converted field to cool down the mechanical motion. Moreover, as the optomechanical coupling is enhanced, the effective squeezing levels of the thermal mechanical state increase, up to about $50 \%$ of squeezing in the figure. This opens the possibility of using the DOMPO to generate squeezed mechanical states.

In Fig. 4 we compare the cooling obtained in our system with the one that would be obtained in a standard sideband cooling scenario. In particular, for a fixed optomechanical coupling $g$, we show $\bar{n}_{\text {eff }}$ and $e^{-2 r_{\text {eff }}}$ as a function of the signal detuning $\delta_{\mathrm{s}}$ and $I_{\mathrm{s}}$, for both the DOMPO and standard sideband cooling. We have studied the latter case by considering the standard scenario consisting of a single driven optical mode interacting with the mechanics, described by the (normalized) quantum Langevin equations

$$
\begin{aligned}
& \frac{d \hat{x}}{d \tau}=\Omega^{2} \hat{p}, \\
& \frac{d \hat{p}}{d \tau}=-\gamma \hat{p}-\hat{x}+2 g \hat{b}_{\mathrm{s}}^{\dagger} \hat{b}_{\mathrm{s}}+\sqrt{\frac{4 \gamma \bar{n}_{\mathrm{th}}}{\Omega}} g_{\mathrm{DC}} \hat{p}_{\mathrm{in}}(\tau), \\
& \frac{d \hat{b}_{\mathrm{s}}}{d \tau}=E-\left(1-\mathrm{i} \delta_{\mathrm{s}}-\mathrm{i} g \hat{x}\right) \hat{b}_{\mathrm{s}}+\sqrt{2} g_{\mathrm{DC}} \hat{b}_{\mathrm{s}, \mathrm{in}}(t),
\end{aligned}
$$

where $E$ is the (normalized) amplitude associated to the laser which drives the optical mode. Note that $g_{\mathrm{DC}}$ appears here just because we are using the same normalization as in the rest of the paper, see Eqs. (5), which is 

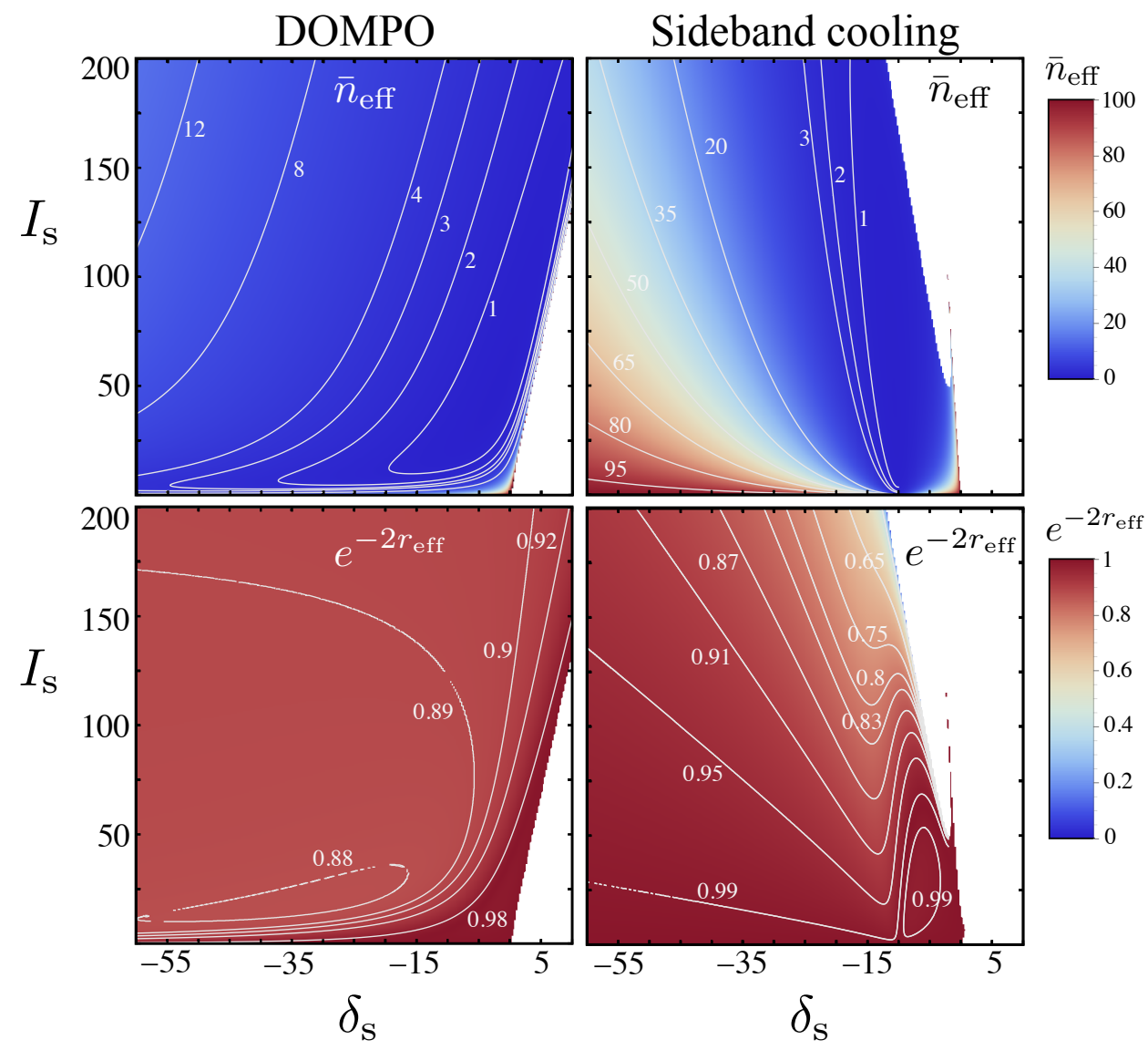

FIG. 4. Density plots of the effective phonon number $\bar{n}_{\text {eff }}$ and mechanical squeezing $e^{-2 r_{\text {eff }}}$ as a function of the signal detuning $\delta_{\mathrm{s}}$ and intensity $I_{\mathrm{s}}$. The rest of parameters have the same values as in Fig. 3, and we have fixed the optomechanical coupling to $g=0.1$. The left panels correspond to the DOMPO, while the right ones correspond to standard sideband cooling. It is to be appreciated how much insensitive the cooling obtained in the DOMPO is to the detuning, which has to be approximately fixed to $-\Omega$ (red sideband) in a standard sideband-cooling scenario. Note that the uncoloured regions correspond to areas of the parameter space where the classical stationary solution is unstable, and hence linearization cannot be applied.

convenient for the sake of comparison; in any case, the final results are independent of this parameter, as can be appreciated from the form of the covariance matrix in Eq. (B4). Collecting the relevant operators in the vector $\hat{\mathbf{b}}=\operatorname{col}\left(\hat{x}, \hat{p}, \hat{b}_{\mathrm{s}}, \hat{b}_{\mathrm{s}}^{\dagger}\right)$, these equations take the linearized form (18) with a noise term $\widehat{\mathbf{f}}(\tau)$ without the pump components, and with a linear stability matrix

$\mathcal{L}=\left(\begin{array}{cccc}0 & \Omega^{2} & 0 & 0 \\ -1 & -\gamma & 2 g \bar{\beta}_{\mathrm{s}}^{*} & 2 g \bar{\beta}_{\mathrm{s}} \\ \mathrm{i} g \bar{\beta}_{\mathrm{s}} & 0 & -\left(1-\mathrm{i} \delta_{\mathrm{s}}-\mathrm{i} g \bar{x}\right) & 0 \\ -\mathrm{i} g \bar{\beta}_{\mathrm{s}}^{*} & 0 & 0 & -\left(1+\mathrm{i} \delta_{\mathrm{s}}+\mathrm{i} g \bar{x}\right)\end{array}\right)$

which is the usual one found in the standard optomechanical case [32, 33]. Following the approach explained above, we obtain the covariance matrix (B4) associated to this problem, and from it, the effective thermal phonon number and effective mechanical squeezing.

The differences in the cooling performance for these two systems can be appreciated in Fig. 4. In particular, it is apparent that the cooling obtained in the DOMPO is less sensitive to the detuning, which in standard sideband cooling needs to be tuned to the red sideband $\delta_{\mathrm{s}} \approx-\Omega$. This insensitivity to the system parameters is something that we have also observed for other parameters, and in other regions of the parameter space, and it constitutes a main difference between the DOMPO and standard sideband optomechanical cooling.

\section{CONCLUSIONS AND OUTLOOK}

In conclusion, in this work we have analyzed the DOMPO from two approximate (but relevant) perspectives: the classical limit and the linearized theory of quantum fluctuations. From a fundamental point of view, such study has been motivated by the question of how does a quantum-correlated field affect the mechanical motion. From a practical viewpoint, the study is timely because it is to be expected that the analyzed model will be experimentally implemented soon, in particular in the form of crystalline whispering gallery mode resonators or 
superconducting circuits.

We have made an exhaustive analysis of the classical phase diagram, which provides highly relevant information prior to the application of more accurate quantum techniques (see [85] for an example in this direction). Our results show that the optomechanical interaction has the effect of introducing new dynamical instabilities not present in the DOPO, as well as changing the location of the instabilities already present in it.

As for the quantum properties, when working above threshold the linearized theory has shown the ability of the quantum-correlated (squeezed) field to cool down the mechanical motion, not only to a regular thermal state, but also to a squeezed thermal state as the optomechanical coupling is enhanced. Moreover, such cooling has been shown to be more insensitive to parameters (most prominently detuning) than the one obtained through standard sideband cooling. Unfortunately, in the threemode problem defined by the DOMPO it is very challenging to get analytical results from the linearization technique, or even to get a conclusive physical picture for the observed phenomenology. Therefore, it will be interesting to apply other techniques which might clarify the physical processes underlying the results presented here. Techniques such as the adiabatic elimination of the optical modes in the master equation of the system might be key to this purpose.

Finally, we emphasize again on the failure of the linearization theory below threshold, where the optomechanical interaction becomes purely nonlinear. This opens an even more interesting venue, since it is a problem which will require more elaborate techniques capable of capturing nongaussian effects. Particularly relevant for us is the c-MoP theory 103 that we recently applied to the DOPO problem 101, which also has the virtue of regularizing the results at the critical points of the classical theory. With the help of such an approach we showed in a recent work [85] that, even below threshold, the down-converted field can cool down the mechanical motion, through a process that we identified by a "cooling by heating" mechanism [104. It will be interesting to analyze then whether this is also the mechanism responsible for the cooling that we observe above threshold in this work.

We have benefited from discussions with Yue Chang, Christoph Marquardt, Eugenio Roldán, Tao Shi, Germán J. de Valcárcel, Joaquín Ruiz-Rivas, Michael J. Hartmann, Florian Marquardt, Vittorio Peano, and J. Ignacio Cirac. S.P.-O. thanks the Theory Division of the MaxPlanck Institute of Quantum Optics for their support and hospitality. F.J. thanks the DFG Collaborative Research Center TRR 109, "Discretization in Geometry and Dynamics". P.D.-S. thanks the German Research Foundation (DFG) for support via the CRC 631 and the grant HA 5593/3-1. C.N.-B. acknowledges the financial support of the Alexander von Humboldt Foundation through its Fellowship for Postdoctoral Researchers.

\section{Appendix A: Details of the stability analysis on the classical solutions}

We provide in this appendix all the details concerning our treatment of the instabilities present in the DOMPO model, which we summarized in Sec. IIIB.

\section{Stability of the trivial solution}

In the case of the trivial stationary solution $\left(\bar{\beta}_{\mathrm{s}}=0\right)$, the linear stability matrix 15 is highly simplified, acquiring in particular a box structure $\mathcal{L}=\mathcal{L}_{\mathrm{m}} \oplus \mathcal{L}_{\mathrm{p}} \oplus \mathcal{L}_{\mathrm{s}}$, where the second block is already in diagonal form

$$
\mathcal{L}_{\mathrm{p}}=\left(\begin{array}{cc}
-\kappa\left(1-\mathrm{i} \delta_{\mathrm{p}}\right) & 0 \\
0 & -\kappa\left(1+\mathrm{i} \delta_{\mathrm{p}}\right)
\end{array}\right),
$$

and its two eigenvalues have negative real part, the first block is given by

$$
\mathcal{L}_{\mathrm{m}}=\left(\begin{array}{cc}
0 & \Omega^{2} \\
-1 & -\gamma
\end{array}\right)
$$

whose eigenvalues $\lambda_{\mathrm{m}}^{( \pm)}=-\left(\gamma \pm \sqrt{\gamma^{2}-4 \Omega^{2}}\right) / 2$ have also negative real part, and finally the last block reads

$$
\mathcal{L}_{\mathrm{s}}=\left(\begin{array}{cc}
-1+\mathrm{i} \delta_{\mathrm{s}} & \bar{\beta}_{\mathrm{p}} \\
\bar{\beta}_{\mathrm{p}}^{*} & -1-\mathrm{i} \delta_{\mathrm{s}}
\end{array}\right),
$$

with eigenvalues

$$
\lambda_{\mathrm{s}}^{( \pm)}=-1 \pm \sqrt{I_{\mathrm{p}}-\delta_{\mathrm{s}}^{2}}
$$

Hence, as commented in the text, we see that $\lambda_{s}^{(+)}$provides a static instability of the trivial solution, located at $I_{\mathrm{p}}=1+\delta_{\mathrm{s}}^{2}$.

\section{Stability of the nontrivial solution}

In the case of the nontrivial solution the $6 \times 6$ linear stability matrix (15) does not have a box structure, and hence their eigenvalues do not have simple analytic expressions. However, we are not as interested in the actual eigenvalues as we are in the points where the real part of some of them becomes zero, since those are the points marking the instabilities, and these points can be found by analyzing the characteristic polynomial of the stability matrix, which we write as $P(\lambda)=\sum_{n=0}^{6} c_{n} \lambda^{n}$. Most of the coefficients $c_{n}\left(I_{\mathrm{s}}, \delta_{\mathrm{s}}, \delta_{\mathrm{p}}, g, \kappa, \gamma, \Omega\right)$ are quite lengthy, and hence we don't show them here, except for the independent one, which can be written as $c_{0}=4 q_{2} I_{\mathrm{s}}+2 q_{1}$, where $q_{1}$ and $q_{2}$ are defined in 12 .

Given the characteristic polynomial, the static instabilities can be found from the condition $P(\lambda=0)=0$, that is, they are located in the region of the parameter space defined by the equation $c_{0}=0$, which in our case 
gives $I_{\mathrm{s}}=I_{\mathrm{s}}^{\mathrm{TP}}$ as mentioned in the text. We can then try to do the same with the Hopf bifurcations, but in that case the expressions are not as easy to handle. It is instructive to first consider the case without optomechanical coupling, $g=0$. In this case the characteristic polynomial can be factorized as $P(\lambda)=P_{\mathrm{DOPO}}(\lambda) P_{\mathrm{m}}(\lambda)$, where $P_{\mathrm{m}}(\lambda)=\lambda^{2}+\gamma \lambda+\Omega^{2}$ is the characteristic polynomial associated to the free mechanical motion (hence showing no instabilities), while $P_{\mathrm{DOPO}}(\lambda)=\sum_{n=0}^{4} d_{n} \lambda^{n}$, with

$$
\begin{aligned}
& d_{0}=\kappa^{2} I_{\mathrm{s}}\left(I_{\mathrm{s}}+2-2 \delta_{\mathrm{p}} \delta_{\mathrm{s}}\right), \\
& d_{1}=2 \kappa\left[I_{\mathrm{s}}+\kappa\left(1+I_{\mathrm{s}}+\delta_{\mathrm{p}}^{2}\right)\right], \\
& d_{2}=\kappa\left[4+2 I_{\mathrm{s}}+\kappa\left(1+\delta_{\mathrm{p}}^{2}\right)\right], \\
& d_{3}=2(1+\kappa), \\
& d_{4}=1,
\end{aligned}
$$

is the characteristic polynomial associated to the optical modes coupled through the parametric down-conversion process, that is, to the DOPO 82,84$]$. The Hopf instabilities are found by locating the points in the parameter space where the eigenvalues become purely imaginary, $\lambda=\mathrm{i} \omega_{\mathrm{HB}}$, where the real parameter $\omega_{\mathrm{HB}}$ is known as the Hopf frequency (providing the frequency of the periodic solution which is born right at the bifurcation). Applying this condition to the DOPO's characteristic polynomial, we get

$$
\begin{aligned}
P_{\mathrm{DOPO}}\left(\lambda=\mathrm{i} \omega_{\mathrm{HB}}\right)= & \left(d_{0}-d_{2} \omega_{\mathrm{HB}}^{2}+d_{4} \omega_{\mathrm{HB}}^{4}\right) \\
& +\mathrm{i} \omega_{\mathrm{HB}}\left(d_{1}-d_{3} \omega_{\mathrm{HB}}^{2}\right)=0
\end{aligned}
$$

the imaginary part of this equation provides us with the Hopf frequency

$$
\omega_{\mathrm{HB}}^{2}=\frac{d_{1}}{d_{3}}=\frac{\kappa\left[I_{\mathrm{s}}+\kappa\left(1+I_{\mathrm{s}}+\delta_{\mathrm{p}}^{2}\right)\right]}{1+\kappa},
$$

which is well defined for every value of the parameters, while the real part of $\mathrm{A} 6$ provides the condition $d_{0} d_{3}^{2}+$ $d_{4} d_{1}^{2}-d_{2} d_{1} d_{3}=0$, which can be solved for $I_{\mathrm{s}}$ analytically, leading to the simple expression of Eq. 16 provided in the text.

In the $g \neq 0$ case the large order of the characteristic polynomial has prevented us from finding simple analytic expressions for the dynamical instabilities of the DOMPO. Let us then pass now to explain how we have dealt with them. Proceeding as in the previous case, the real and imaginary parts of $P\left(\lambda=\mathrm{i} \omega_{\mathrm{HB}}\right)$ provide us with two coupled equations

$$
\begin{aligned}
c_{0}-c_{2} \omega_{\mathrm{HB}}^{2}+c_{4} \omega_{\mathrm{HB}}^{4}-c_{6} \omega_{\mathrm{HB}}^{6} & =0, \\
\omega_{\mathrm{HB}}\left(c_{1}-c_{3} \omega_{\mathrm{HB}}^{2}+c_{5} \omega_{\mathrm{HB}}^{4}\right) & =0 .
\end{aligned}
$$

We can see that $\omega_{\mathrm{HB}}=0$ and $c_{0}=0$ is a solution of the equations, that is, they contain the pitchfork bifurcation, what is not surprising since they are general and valid for any type of instability. Now, for $\omega_{\mathrm{HB}} \neq 0$, we can proceed as follows. The second equation $\mathrm{A} 8 \mathrm{~b}$ can be solved for the Hopf frequency as

$$
\omega_{\mathrm{HB}, \pm}^{2}=\frac{c_{3} \pm \sqrt{c_{3}^{2}-4 c_{1} c_{5}}}{2 c_{5}} ;
$$

these solutions can be introduced in A8a, but unfortunately the resulting equations do not allow to find a simple analytic solution for $I_{\mathrm{s}}$. However, a symbolic program such as Mathematica allows us to find analytic solutions, provided that we write the equation as a more manageable polynomial. In particular, let us write $\omega_{\mathrm{HB}, \pm}^{2}=l \pm r$ with $l=c_{3} / 2 c_{5}$ and $r=\sqrt{c_{3}^{2}-4 c_{1} c_{5}} / 2 c_{5}$, which allows us to rewrite A8a as

$$
\begin{aligned}
c_{0}-c_{2} l+c_{4}\left(l^{2}+r^{2}\right) & -c_{6}\left(l^{3}+3 l r^{2}\right) \\
& = \pm r\left[c_{2}-2 c_{4} l+c_{6}\left(3 l^{2}+r^{2}\right)\right] .
\end{aligned}
$$

The square of this expression provides a sixth order polynomial equation for $I_{\mathrm{s}}$, whose solutions can be efficiently handled by a symbolic program. Note that by taking the square of the previous equation, we are indeed introducing extra fictitious solutions for $I_{\mathrm{s}}$, but we have checked that these extra solutions are always complex, and hence they do not provide anything which could be interpreted as instabilities. This procedure has allowed us to make an exhaustive numerical analysis of the Hopf instabilities for $g \neq 0$, of which we have shown a characteristic example in Fig. 2 .

\section{Appendix B: Evaluation of the covariance matrix and the effective mechanical parameters}

In this section we explain a route to find the covariance matrix of the DOMPO directly from the linearized quantum Langevin equations (18). We also prove expression 25 for the effective mechanical thermal phonon number and squeezing.

In order to find the covariance matrix $V$ we proceed as follows. First, we find the left-eigenvectors of the linear stability matrix. These can be computed as the eigenvectors of its transpose, $\left\{\mathcal{L}^{T} \mathbf{w}_{j}=\lambda_{j} \mathbf{w}_{j}\right\}_{j=1,2, \ldots, 6}$, from which we build the matrix $\mathcal{W}=\operatorname{col}\left(\mathbf{w}_{1}^{T}, \mathbf{w}_{2}^{T}, \ldots, \mathbf{w}_{6}^{T}\right)$, as well as the diagonal matrix of eigenvalues $\Lambda=$ $\operatorname{diag}\left(\lambda_{1}, \lambda_{2}, \ldots, \lambda_{6}\right)$. With these definitions, we have $\mathcal{W} \mathcal{L}=\Lambda \mathcal{W}$. Hence, applying $\mathcal{W}$ on the left of the linearized Langevin equations (18), and defining the vector $\hat{\mathbf{c}}(\tau)=\mathcal{W} \delta \hat{\mathbf{b}}(\tau)$, we get a set of uncoupled linear equations for its components, leading to the solution

$$
\hat{\mathbf{c}}(\tau)=\sqrt{2} g_{\mathrm{DC}} \int_{0}^{\tau} d \tau^{\prime} e^{\Lambda\left(\tau-\tau^{\prime}\right)} \mathcal{W} \widehat{\mathbf{f}}\left(\tau^{\prime}\right),
$$

in the asymptotic limit $\tau \gg \max _{j=1,2, \ldots, 6} \operatorname{Re}\left\{\lambda_{j}\right\}^{-1}$. It is then straightforward to compute the corresponding correlation matrix $\mathcal{C}$, with elements $C_{j l}(\tau)=\left\langle\hat{c}_{j}(\tau) \hat{c}_{l}(\tau)\right\rangle$, which in the asymptotic limit read

$$
C_{j l}=-2 g_{\mathrm{DC}}^{2} \frac{\left(\mathcal{W} \mathcal{M} \mathcal{W}^{T}\right)_{j l}}{\lambda_{j}+\lambda_{l}}
$$


where the matrix $\mathcal{M}$ is defined in 20.

On the other hand, the quadrature vector $\hat{\mathbf{r}}$ is related to the vector $\hat{\mathbf{b}}$ by $\hat{\mathbf{r}}=\mathcal{R} \hat{\mathbf{b}}$, where $\mathcal{R}=\mathcal{R}_{\mathrm{m}} \oplus \mathcal{R}_{\mathrm{p}} \oplus \mathcal{R}_{\mathrm{s}}$, with

$$
\begin{aligned}
& \mathcal{R}_{\mathrm{m}}=\frac{1}{g_{\mathrm{DC}}}\left(\begin{array}{cc}
1 / \sqrt{\Omega} & 0 \\
0 & \sqrt{\Omega}
\end{array}\right), \\
& \mathcal{R}_{\mathrm{p}}=\frac{1}{g_{\mathrm{DC}} \sqrt{\kappa}}\left(\begin{array}{cc}
1 & 1 \\
-\mathrm{i} & \mathrm{i}
\end{array}\right), \\
& \mathcal{R}_{\mathrm{s}}=\frac{1}{g_{\mathrm{DC}}}\left(\begin{array}{cc}
1 & 1 \\
-\mathrm{i} & \mathrm{i}
\end{array}\right)
\end{aligned}
$$

and hence, its fluctuations can be written as $\delta \hat{\mathbf{r}}(\tau)=$ $\mathcal{R} \mathcal{W}^{-1} \hat{\mathbf{c}}(\tau)$, leading to the final form of the covariance matrix in the asymptotic limit

$$
V=\mathcal{R} \mathcal{W}^{-1}\left(\mathcal{C}+\mathcal{C}^{T}\right) \mathcal{W}^{-1 T} \mathcal{R}^{T}
$$

This is the expression that we have used to compute the Gaussian steady state of the system, which can be efficiently evaluated numerically for any value of the parameters, since it just requires diagonalizing the $6 \times 6$ matrix $\mathcal{L}^{T}$.
Let us now pass to derive the relation between the effective mechanical parameters and the elements of the mechanical covariance matrix. In order to find it, we just need to realize that the thermal state is a Gaussian state with covariance matrix $V_{\mathrm{th}}\left(\bar{n}_{\mathrm{eff}}\right)=\left(2 \bar{n}_{\mathrm{eff}}+1\right) \mathcal{I}_{2 \times 2}$, where $\mathcal{I}_{2 \times 2}$ is the $2 \times 2$ identity matrix, while the squeezing operator simply acts as the symplectic transformation $\mathcal{S}\left(r_{\text {eff }}\right)=\operatorname{diag}\left(e^{-r_{\text {eff }}}, e^{r_{\text {eff }}}\right)$ in the space of covariance matrices [15, 16. Hence, the Gaussian state corresponding to (24) has a diagonal covariance matrix

$$
\begin{aligned}
\tilde{V}_{\mathrm{m}}\left(\bar{n}_{\mathrm{eff}}, r_{\mathrm{eff}}\right) & =\mathcal{S}\left(r_{\mathrm{eff}}\right) V_{\mathrm{th}}\left(\bar{n}_{\mathrm{eff}}\right) \mathcal{S}^{T}\left(r_{\mathrm{eff}}\right) \\
& =\left(2 \bar{n}_{\mathrm{eff}}+1\right) \operatorname{diag}\left(e^{-2 r_{\text {eff }}}, e^{2 r_{\text {eff }}}\right) .
\end{aligned}
$$

Therefore, the phase-shift $\hat{R}(\theta)$ applied to $\hat{\rho}_{\mathrm{m}}$ in Eq. 24 is nothing but the rotation that diagonalizes $V_{\mathrm{m}}$, turning it into

$$
\tilde{V}_{\mathrm{m}}=\operatorname{diag}\left(V_{-}, V_{+}\right)
$$

where the eigenvalues of $V_{\mathrm{m}}$ are given by $V_{\mp}=$ $\operatorname{tr}\left\{V_{\mathrm{m}}\right\} / 2 \mp \sqrt{\operatorname{tr}\left\{V_{\mathrm{m}}\right\}^{2} / 4-\operatorname{det}\left\{V_{\mathrm{m}}\right\}}$. In other words, $\theta$ is the angle in phase space that captures the direction in which squeezing is applied to the mechanical motion. Matching the expressions (B5) and (B6) for the diagonal forms of $\tilde{V}_{\mathrm{m}}$ provides the expressions 25 written in the text.
[1] R. W. Boyd, Nonlinear Optics (Academic, 2003).

[2] P. Meystre and D. F. Walls (eds.), Nonclassical Effects in Quantum Optics (American Institute of Physics, 1991).

[3] C. Navarrete-Benlloch, arXiv:1504.05917

[4] H. J. Carmichael, Statistical Methods in Quantum Optics 2, Springer Verlag, Berlin (2008).

[5] Y. Takeno, M. Yukawa, H. Yonezawa, and A. Furusawa, Opt. Express 15, 4321-4327 (2007).

[6] H. Vahlbruch, M. Mehmet, S. Chelkowski, B. Hage, A. Franzen, N. Lastzka, S. Gossler, K. Danzmann, and R. Schnabel, Phys. Rev. Lett. 100, 033602 (2008).

[7] T. Eberle, S. Steinlechner, J. Bauchrowitz, V. Handchen, H. Vahlbruch, M. Mehmet, H. Muller-Ebhardt, and R. Schnabel, Phys. Rev. Lett. 104, 251102 (2010).

[8] M. Mehmet, H. Vahlbruch, N. Lastzka, K. Danzmann, and R. Schnabel, Phys. Rev. A 81, 013814 (2010).

[9] The LIGO Scientific Collaboration, Nat. Phys. 7, 962 (2011).

[10] K. Goda, O. Miyakawa, E. E. Mikhailov, S. Saraf, R. Adhikari, K. McKenzie, R. Ward, S. Vass, A. J. Weinstein, and N. Mavalvala, Nat. Phys. 4, 472-476 (2008).

[11] H. Vahlbruch, S. Chelkowski, B. Hage, A. Franzen, K. Danzmann, and R. Schnabel, Phys. Rev. Lett. 95, 211102 (2005).

[12] N. Treps, N. Grosse, W. P. Bowen, C. Fabre, H.-A. Bachor, and P. K. Lam, Science 301, 940-943 (2003).

[13] N. Treps, U. Andersen, B. Buchler, P. K. Lam, A. Maître, H.-A. Bachor, and C. Fabre, Phys. Rev. Lett. 88, 203601 (2002).
[14] S. L. Braunstein and P. van Loock, Rev. Mod. Phys. 77, 513-577 (2005).

[15] C. Weedbrook, S. Pirandola, R. García-Patron, N. J. Cerf, T. C. Ralph, J. H. Shapiro, and S. Lloyd, Rev. Mod. Phys. 84, 621-669 (2012).

[16] C. Navarrete-Benlloch, An Introduction to the Formalism of Quantum Information with Continuous Variables (Morgan \& Claypool Publishers and IOP Publishing, 2015).

[17] T. J. Kippenberg and K. J. Vahala, Opt. Exp. 15, 17172 (2007).

[18] F. Marquardt and S. M. Girvin, Physics 2, 40 (2009).

[19] P. Meystre, Annalen der Physik 525, 215 (2013).

[20] M. Aspelmeyer, T. J. Kippenberg, and F. Marquardt, Rev. Mod. Phys. 86, 1391 (2014).

[21] S. Gigan, H. R. Böhm, M. Paternostro, F. Blaser, G. Langer, J. B. Hertzberg, K. C. Schwab, D. Bäuerle, M. Aspelmeyer, and A. Zeilinger, Nature 444, 67 (2006).

[22] O. Arcizet, P.-F. Cohadon, T. Briant, M. Pinard, and A. Heidmann, Nature 444, 71 (2006).

[23] A. Schliesser, P. Del'Haye, N. Nooshi, K. J. Vahala, and T. J. Kippenberg, Phys. Rev. Lett. 97, 243905 (2006).

[24] T. Corbitt, Y. Chen, E. Innerhofer, H. Müller-Ebhardt, D. Ottaway, H. Rehbein, D. Sigg, S. Whitcomb, C. Wipf, and N. Mavalvala, Phys. Rev. Lett. 98, 150802 (2007).

[25] J. D. Thompson, B. M. Zwickl, A. M. Jayich, F. Marquardt, S. M. Girvin, and J. G. E. Harris, Nature 452, 72 (2008).

[26] D. J. Wilson, C. A. Regal, S. B. Papp, and H. J. Kimble, 
Phys. Rev. Lett. 103, 207204 (2009)

[27] J. D. Teufel, T. Donner, D. Li, J. W. Harlow, M. S. Allman, K. Cicak, A. J. Sirois, J. D. Whittaker, K. W. Lehnert, and R. W. Simmonds, Nature 475, 359 (2011).

[28] J. Chan, T. P. Mayer Alegre, A. H. Safavi-Naeini, J. T. Hill1, A. Krause1, S. Gröblacher, M. Aspelmeyer, and O. Painter, Nature 478, 89 (2011).

[29] M. Karuza, C. Molinelli, M. Galassi, C. Biancofiore, R. Natali, P. Tombesi, G. Di Giuseppe, and D. Vitali, New J. Phys. 14, 095015 (2012).

[30] M. Underwood, D. Mason, D. Lee, H. Xu, L. Jiang, A. B. Shkarin, K. Børkje, S. M. Girvin, J. G. E. Harris, Phys. Rev. A 92, 061801(R).

[31] I. Wilson-Rae, N. Nooshi, W. Zwerger, and T. J. Kippenberg, Phys. Rev. Lett. 99, 093901 (2007).

[32] F. Marquardt, J. P. Chen, A. A. Clerk, and S. M. Girvin, Phys. Rev. Lett. 99, 093902 (2007).

[33] C. Genes, D. Vitali, P. Tombesi, S. Gigan, and M. Aspelmeyer, Phys. Rev. A 77, 033804 (2008).

[34] W. Marshall, C. Simon, R. Penrose, and D. Bouwmeester, Phys. Rev. Lett. 91, 130401 (2003).

[35] D. Kleckner, I. Pikovski, E. Jeffrey, L. Ament, E. Eliel, J. van den Brink, and D. Bouwmeester, New J. Phys. 10, 095020 (2008).

[36] O. Romero-Isart, A. C. Pflanzer, F. Blaser, R. Kaltenbaek, N. Kiesel, M. Aspelmeyer, and J. I. Cirac, Phys. Rev. Lett. 107, 020405 (2011).

[37] O. Romero-Isart, Phys. Rev. A 84, 052121 (2012).

[38] C. Fabre, Phys. Rev. A 49, 1337 (1994).

[39] S. Mancini and P. Tombesi, Phys. Rev. A 49, 4055 (1994).

[40] D. W. C. Brooks, T. Botter, S. Schreppler, T. P. Purdy, N. Brahms, and D. M. Stamper-Kurn, Nature 488, 476 (2012).

[41] A. H. Safavi-Naeini, S. Gröblacher, J. T. Hill, J. Chan, M. Aspelmeyer, and O. Painter, Nature 500, 185 (2013).

[42] S. Weis, R. Rivière, S. Deléglise, E. Gavartin, O. Arcizet, A. Schliesser, and T. J. Kippenberg, Science 330 , 1520 (2010)

[43] A. H. Safavi-Naeini, T. P. M. Alegre, J. Chan, M. Eichenfield, M. Winger, Q. Lin, J. T. Hill, D. E. Chang, and O. Painter, Nature 472, 69 (2011).

[44] J. D. Teufel, D. Li, M. S. Allman, K. Cicak, A. J. Sirois, J. D. Whittaker, and R. W. Simmonds, Nature 471, 204 (2011).

[45] F. Massel, S. U. Cho, J.-M. Pirkkalainen, P. J. Hakonen, T.T.Heikkilä, and M. A. Sillanpää, Nat. Commun. 3, 987 (2012)

[46] M. Karuza, C. Biancofiore, M. Bawaj, C. Molinelli, M. Galassi, R. Natali, P. Tombesi, G. Di Giuseppe, and D. Vitali, Phys. Rev. A 88, 013804 (2013).

[47] P. Rabl, Phys. Rev. Lett. 107, 063601 (2011).

[48] K. Stannigel, P. Rabl, A. S. Sørensen, P. Zoller, and M. D. Lukin, Phys. Rev. Lett. 105, 220501 (2010).

[49] A. H Safavi-Naeini and O. Painter, New J. Phys. 13, 013017 (2011).

[50] C. A. Regal and K. W. Lehnert, J. Phys.: Conference Series 264, 012025 (2011)

[51] J. M. Taylor, A. S. Sørensen, C. M. Marcus, and E. S. Polzik, Phys. Rev. Lett. 107, 273601 (2011).

[52] Y.-D. Wang and A. A. Clerk, Phys. Rev. Lett. 108, 153603 (2012).

[53] Y.-D. Wang and A. A. Clerk, New J. Phys. 14, 105010 (2012).
[54] Sh. Barzanjeh, M. Abdi, G. J. Milburn, P. Tombesi, and D. Vitali, Phys. Rev. Lett. 109, 130503 (2012).

[55] J. Bochmann, A. Vainsencher, D. D. Awschalom and A. N. Cleland, Nature Phys. 9, 712 (2013).

[56] T. Bagci, A. Simonsen, S. Schmid, L. G. Villanueva, E. Zeuthen, J. Appel, J. M. Taylor, A. Sørensen, K. Usami, A. Schliesser, and E. S. Polzik, Nature 507, 81 (2014).

[57] R. W. Andrews, R. W. Peterson, T. P. Purdy, K. Cicak, R. W. Simmonds, C. A. Regal, and K. W. Lehnert, Nature Phys. 10, 321 (2014).

[58] A. Schliesser, Cavity Optomechanics and Optical Frequency Comb Generation with Silica WhisperingGallery-Mode Microresonators (PhD thesis, LudwigMaximilians-Universität München, 2009).

[59] T. A. Palomaki, J. W. Harlow, J. D. Teufel, R. W. Simmonds, and K. W. Lehnert, Nature 495, 210 (2013).

[60] S. Huang and G. S. Agarwal, Phys. Rev. A 79, 013821 (2009).

[61] S. Huang and G. S. Agarwal, Phys. Rev. A 80, 033807 (2009).

[62] V. Peano, H. G. L. Schwefel, Ch. Marquardt, and F. Marquardt, Phys. Rev. Lett. 115, 243603 (2015).

[63] G. S. Agarwal and S. Huang, arXiv:1602.02214

[64] X.-Y. Lü, Y. Wu, J. R. Johansson, H. Jing, J. Zhang, and F. Nori, Phys. Rev. Lett. 114, 093602 (2015).

[65] A. Xuereb, M. Barbieri, and M. Paternostro, Phys. Rev. A 86, 013809 (2012).

[66] V. S. Ilchenko, A. A. Savchenkov, A. B. Matsko, and L. Maleki, J. Opt. Soc. Am. B 20, 333 (2003).

[67] V. S. Ilchenko, A. A. Savchenkov, A. B. Matsko, and L. Maleki, Phys. Rev. Lett. 92, 043903 (2004).

[68] A. A. Savchenkov, A. B. Matsko, M. Mohageg, D. V. Strekalov, and L. Maleki, Opt. Lett. 32, 157 (2007).

[69] J. U. Fürst, D. V. Strekalov, D. Elser, M. Lassen, U. L. Andersen, C. Marquardt, and G. Leuchs, Phys. Rev. Lett. 104, 153901 (2010).

[70] J. U. Fürst, D. V. Strekalov, D. Elser, A. Aiello, U. L. Andersen, Ch. Marquardt, and G. Leuchs, Phys. Rev. Lett. 105, 263904 (2010).

[71] J. Hofer, A. Schliesser, and T. J. Kippenberg, Phys. Rev, A 82, 031804(R) (2010).

[72] J. U. Fürst, D. V. Strekalov, D. Elser, A. Aiello, U. L. Andersen, Ch. Marquardt, and G. Leuchs, Phys. Rev. Lett. 106, 113901 (2011).

[73] T. Beckmann, H. Linnenbank, H. Steigerwald, B. Sturman, D. Haertle, K. Buse, and I. Breunig, Phys. Rev. Lett. 106, 143903 (2011).

[74] C. S. Werner, T. Beckmann, K. Buse, and I. Breunig, Opt. Lett. 37, 4224 (2012).

[75] M. Förtsch, J. U. Fürst, C. Wittmann, D. Strekalov, A. Aiello, M. V. Chekhova, C. Silberhorn, G. Leuchs, and C. Marquardt, Nature Commun. 4, 1818 (2013).

[76] C. Marquardt, D. Strekalov, J. Fürst, M. Förtsch, and G. Leuchs, Opt. Phot. News 24, 38 (2013).

[77] M. Förtsch, G. Schunk, J. U. Fürst, D. Strekalov, T. Gerrits, M. J. Stevens, F. Sedlmeir, H. G. L. Schwefel, S. W. Nam, G. Leuchs, and C. Marquardt, Phys. Rev. A 91, 023812 (2015).

[78] M. Förtsch, T. Gerrits, M. J. Stevens, D. Strekalov, G. Schunk, J. U. Fürst, U. Vogl, F. Sedlmeir, H. G. L. Schwefel, G. Leuchs, S. W. Nam, and C. Marquardt, J. Opt. 17, 065501 (2015).

[79] Z. Leghtas, S. Touzard, I. M. Pop, A. Kou, B. Vlastakis, A. Petrenko, K. M. Sliwa, A. Narla, S. Shankar, M. J. 
Hatridge, M. Reagor, L. Frunzio, R. J. Schoelkopf, M. Mirrahimi, M. H. Devoret, Science 347, 853 (2015).

[80] P. D. Nation, J. Suh, and M. P. Blencowe, arXiv:1507.00115.

[81] S. Gigan, L. Lopez, V. Delaubert, N. Treps, C. Fabre, A. Maitre, J. Mod. Opt. 53, 809 (2006).

[82] L. Lugiato, C. Oldano, C. Fabre, E. Giacobino, and R. J. Horowicz, Il Nuovo Cimento 10, 959 (1988).

[83] N. P. Pettiaux, R.-D. Li, and P. Mandel, Optics Commun. 72, 256 (1989).

[84] C. Fabre, E. Giacobino, A. Heidmann, L. Lugiato, S. Reynaud, M. Valdacchino, and W. Kaige, Quantum Opt. 2, 159 (1990).

[85] P. Degenfeld-Schonburg, M. Abdi, M. J. Hartmann, and C. Navarrete-Benlloch, Phys. Rev. A, in press.

[86] D.F. Walls and G. Milburn, Quantum Optics, Springer (2007).

[87] C. W. Gardiner and P. Zoller, Quantum Noise, Springer Verlag (2004).

[88] L. M. Narducci and N. B. Abraham, Laser Physics and Laser Instabilities (World Scientific, Singapore, 1988).

[89] C. Navarrete-Benlloch, E. Roldán, Y. Chang, and T. Shi, Optics Express 22, 024010 (2014).

[90] P. D. Drummond, K. J. McNeil, and D. F. Walls, J. Mod. Opt. 28, 211 (1981).

[91] L. A. Lugiato and G. Strini, Opt. Commun. 41, 67
(1981).

[92] M. J. Collett and C. W. Gardiner, Phys. Rev. A 30, 1386 (1984).

[93] P. Kinsler, M. Fernée, and P. D. Drummond, Phys. Rev. A 48, 3310 (1993).

[94] P. Kinsler and P. D. Drummond, Phys. Rev. A 52, 783 (1995).

[95] P. D. Drummond, K. Dechoum, and S. Chaturvedi, Phys. Rev. A 65, 033806 (2002).

[96] S. Chaturvedi and P. D. Drummond, Eur. Phys. J. B 8, 251 (1999).

[97] D. T. Pope, P. D. Drummond, and S. Chaturvedi, Phys. Rev. A 62, 042108 (2000).

[98] C. J. Mertens, T. A. B. Kennedy, and S. Swain Phys. Rev. Lett., 71, 2014 (1993).

[99] C. J. Mertens, T. A. B. Kennedy, and S. Swain Phys. Rev. A, 48, 2374 (1993).

[100] O. Veits and M. Fleischhauer, Phys. Rev. A 55, 3059 (1997).

[101] P. Degenfeld-Schonburg, C. Navarrete-Benlloch, and M. J. Hartmann, Phys. Rev. A 91, 053850 (2015).

[102] P. D. Drummond and C. W. Gardiner, J. Phys. A: Math. Gen. 13, 2353 (1980).

[103] P. Degenfeld-Schonburg and M. J. Hartmann, Phys. Rev. B 89, 245108 (2014).

[104] A. Mari and J. Eisert, Phys. Rev. Lett. 108, 120602 (2012). 